\title{
Editorial: Epigenetic and Transcriptional Dysregulations in Cancer and Therapeutic Opportunities
}

\author{
Rais A. Ansari ${ }^{1}$ and Ata Abbas ${ }^{2,3 *}$ \\ ${ }^{1}$ Department of Pharmaceutical Sciences, Nova Southeastern University, Fort Lauderdale, FL, United States, ${ }^{2}$ Division of \\ Hematology and Oncology, Department of Medicine, Case Western Reserve University, Cleveland, OH, United States, ${ }^{3}$ Case \\ Comprehensive Cancer Center, Case Western Reserve University School of Medicine, Cleveland, OH, United States
}

Keywords: epigenetics, transcription, transcriptional dysregultaion, cancer, cancer therapies

Editorial on the Research Topic

Epigenetic and Transcriptional Dysregulations in Cancer and Therapeutic Opportunities

Aberrations in epigenetic regulation at various levels, including DNA methylation, chromatin architecture, and regulatory RNAs, are often associated with, and significantly contribute in most carcinogenesis (Jones and Baylin, 2007; Baylin and Jones, 2016). Transcriptional dysregulation is another hallmark of nearly all kinds of cancers (Hanahan, 2022). Gene transcription is a complex process and highly regulated at various stages as well as at the post-transcriptional level (Corbett, 2018; Roeder, 2019). Epigenetic and transcriptional events often influence each other, e.g., DNA methylation and histone modifications regulate gene transcription, and transcriptional processes can modify chromatin architectures (Bonasio et al., 2010; Gibney and Nolan, 2010). Increasing numbers

\section{OPEN ACCESS}

Edited and reviewed by: Michael E. Symonds, University of Nottingham, United Kingdom

${ }^{*}$ Correspondence: Ata Abbas ata.abbas@case.edu

Specialty section:

This article was submitted to Epigenomics and Epigenetics,

a section of the journal

Frontiers in Genetics

Received: 18 January 2022 Accepted: 07 February 2022 Published: 22 February 2022

Citation: Ansari RA and Abbas A (2022) Editorial: Epigenetic and Transcriptional Dysregulations in

Cancer and

Therapeutic Opportunities.

Front. Genet. 13:857380.

doi: 10.3389/fgene.2022.857380 of evidence suggest epigenetic and transcriptional dysregulations play vital roles in carcinogenesis, including metastasis, aggressiveness, and recurrence of malignancies (Bradner et al., 2017; Hanahan, 2022). An in-depth understanding of both epigenetic and transcriptional processes and alterations in their regulations are needed to better understand tumor pathobiology and to improve clinical management (Figure 1). Epigenetic and transcriptional dysregulations also confer therapeutic vulnerabilities and remarkably, offer novel biomarkers and therapeutic targets (Gonda and Ramsay, 2015; Cheng et al., 2019; Lu et al., 2020; Vervoort et al., 2021).

Genome-wide transcriptional control is often dysregulated in cancer. In this research topic, Parrello et al. discussed the possibility of targeting factors that control global transcriptional regulation. Li et al. discussed the dual roles of CBX7, a component of polycomb repressive complex, in cancer where it can either help in cancer progression by downregulating tumor suppressor genes or help cancer suppression by modulating cell cycle related proteins. CBX7 interacts with various regulatory RNAs, including micro RNAs, long non-coding RNAs, circular RNAs. Regulatory RNAs play a significant role in carcinogenesis, including chemo-resistance (Lan et al., Gareev et al., and Zhang et al.). Sun et al. showed that plasma-derived exosomal micro RNA, miR-2276-5p in glioma patients could serve as a potential diagnostic and prognostic marker. Transcriptomics based gene signatures are emerging as promising biomarkers in cancer (Jin et al. and Fang et al.).

Epigenetic landscape is altered in cancer cells that results in transcriptional dysregulation. Various dietary components have ability to modulate epigenetic aberration (Fatima et al. and Raina et al.). Abbas et al. reported that maternal diet rich in omega-3 fatty acid can reprogram epigenetic and transcriptomic landscapes in F1 generation mice and provide resistance to breast cancer development. $\mathrm{Pu}$ et al. reported that methylation profiles of zinc finger genes, specially ESR1 and ZNF132, could be potential biomarkers for the early diagnosis of colorectal cancer patients carrying KRAS mutations. Another study by Gua et al. showed that APOA1 gene is downregulated by DNA methylation in 


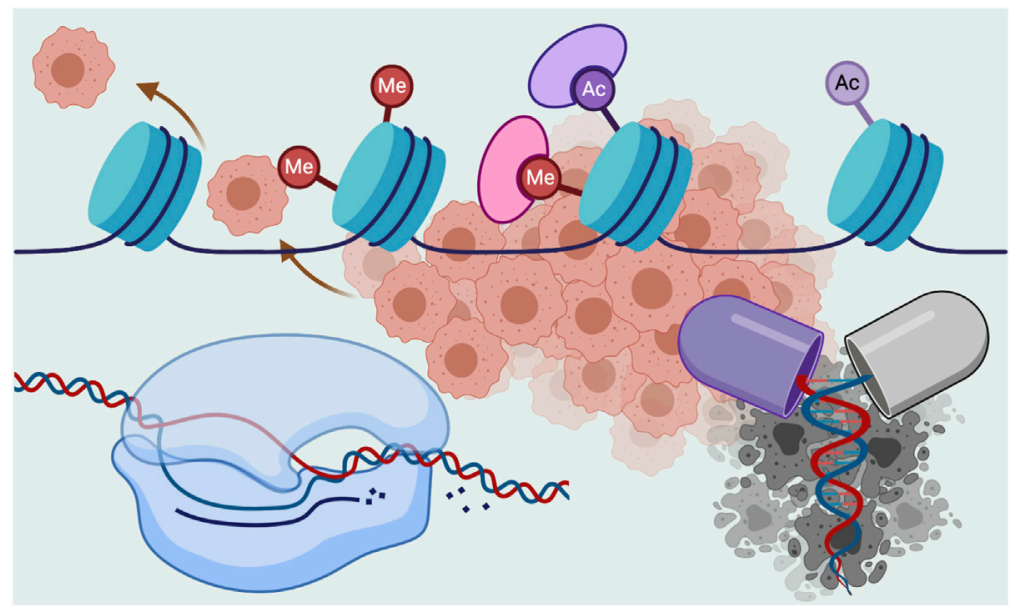

FIGURE 1 | Alteration of epigenetic and transcriptional processes often contribute in carcinogenic events. These alterations can also serve as novel therapeutic targets and biomarkers (created with BioRender.com).

hepatocellular carcinoma that could be a potential biomarker to predict survival. Role of NTPCR in epithelial ovarian cancer (Shang et al.) and FGFR1-GLI1 axis as a potential therapeutic target in breast cancer (Riaz et al.) were also reported.

Gazova et al. used CRISPR-Cas9 to generate homozygous inactivating mutation in USP16 gene using leukemia cell line and studied how these cells adapt to the extreme selection pressure through compensatory pathways. Authors also cautioned targeting USP16 in leukemia as cancer could develop resistant to USP16 inhibitors. A timely review by Amir et al. discussed the usefulness of combination therapy of tyrosine kinase inhibitors with epigenetic drugs in chronic myeloid leukemia. Leszczynska et al. reviewed the emerging therapeutic approaches

\section{REFERENCES}

Baylin, S. B., and Jones, P. A. (2016). Epigenetic Determinants of Cancer. Cold Spring Harb Perspect. Biol. 8, a019505. doi:10.1101/cshperspect.a019505

Bonasio, R., Tu, S., and Reinberg, D. (2010). Molecular Signals of Epigenetic States. Science 330, 612-616. doi:10.1126/science.1191078

Bradner, J. E., Hnisz, D., and Young, R. A. (2017). Transcriptional Addiction in Cancer. Cell 168, 629-643. doi:10.1016/j.cell.2016.12.013

Cheng, Y., He, C., Wang, M., Ma, X., Mo, F., Yang, S., et al. (2019). Targeting Epigenetic Regulators for Cancer Therapy: Mechanisms and Advances in Clinical Trials. Signal. Transduction Targeted Ther. 4, 62. doi:10.1038/ s41392-019-0095-0

Corbett, A. H. (2018). Post-transcriptional Regulation of Gene Expression and Human Disease. Curr. Opin. Cell Biol. 52, 96-104. doi:10.1016/j.ceb.2018.02.011

Gibney, E. R., and Nolan, C. M. (2010). Epigenetics and Gene Expression. Heredity 105, 4-13. doi:10.1038/hdy.2010.54

Gonda, T. J., and Ramsay, R. G. (2015). Directly Targeting Transcriptional Dysregulation in Cancer. Nat. Rev. Cancer 15, 686-694. doi:10.1038/nrc4018

Hanahan, D. (2022). Hallmarks of Cancer: New Dimensions. Cancer Discov. 12, 31-46. doi:10.1158/2159-8290.cd-21-1059

Jones, P. A., and Baylin, S. B. (2007). The Epigenomics of Cancer. Cell 128, 683-692. doi:10.1016/j.cell.2007.01.029

Lu, Y., Chan, Y. T., Tan, H. Y., Li, S., Wang, N., and Feng, Y. (2020). Epigenetic Regulation in Human Cancer: the Potential Role of Epi- against pediatric high-grade gliomas, particularly those having mutations in genes coding for histone 3 variants that result in substitution of lysine at 27 to methionine.

In conclusion, epigenetic aberration and transcriptional homeostasis disruptions are associated with cancer. In-depth understanding of these processes and their interdependencies is needed to better understand carcinogenesis and to develop novel and effective therapeutic approaches.

\section{AUTHOR CONTRIBUTIONS}

RAA and AA wrote the manuscript.

Drug in Cancer Therapy. Mol. Cancer 19, 79. doi:10.1186/s12943-02001197-3

Roeder, R. G. (2019). 50+ Years of Eukaryotic Transcription: an Expanding Universe of Factors and Mechanisms. Nat. Struct. Mol. Biol. 26, 783-791. doi:10.1038/s41594-019-0287-x

Vervoort, S. J., Devlin, J. R., Kwiatkowski, N., Teng, M., Gray, N. S., and Johnstone, R. W. (2021). Targeting Transcription Cycles in Cancer. Nat. Rev. Cancer 22, 5-24. doi:10.1038/s41568-021-00411-8

Conflict of Interest: The authors declare that the research was conducted in the absence of any commercial or financial relationships that could be construed as a potential conflict of interest.

Publisher's Note: All claims expressed in this article are solely those of the authors and do not necessarily represent those of their affiliated organizations, or those of the publisher, the editors and the reviewers. Any product that may be evaluated in this article, or claim that may be made by its manufacturer, is not guaranteed or endorsed by the publisher.

Copyright (C) 2022 Ansari and Abbas. This is an open-access article distributed under the terms of the Creative Commons Attribution License (CC BY). The use, distribution or reproduction in other forums is permitted, provided the original author(s) and the copyright owner(s) are credited and that the original publication in this journal is cited, in accordance with accepted academic practice. No use, distribution or reproduction is permitted which does not comply with these terms. 